\title{
Ser niña, indígena y migrante. Curso de vida y agencia en contextos sociales signados por la violencia de género y la desigualdad. El caso de una niña tsotsil originaria de los Altos de Chiapas ${ }^{1}$
}

\section{Sarai Miranda² CONACYT ECOSUR}

\section{Artículo científico}

Material original autorizado para su primera publicación en el Journal de Ciencias Sociales, Revista Académica de la Facultad de Ciencias Sociales de la Universidad de Palermo.

\section{Recepción: 13-03-2018}

Aceptación: 17-07-2018

Resumen: En México, el estado de Chiapas es la entidad que ha mostrado mayores atrasos sociales desde larga data. La mayoría de la población que habita a lo largo del territorio chiapaneco cuenta con escaso acceso a las condiciones mínimas para el logro del bienestar, pero además experimenta día a día la persistencia de la discriminación por la vía de la clase, el género y la etnia, lo que abona a la profundización de la vulnerabilidad social. Este artículo versa sobre la trayectoria de una niña indígena tsotsil de la región de los Altos de Chiapas, quien, en un contexto familiar y comunitario marcado por la desigualdad y la violencia, ha logrado hacer uso de sus recursos individuales para cambiar el curso de su vida. El objetivo general es describir las diversas estrategias que la niña despliega a lo largo de su vida e identificar los recursos que le han permitido ejercer la agencia para salir de algunas situaciones de violencia y discriminación. El acercamiento a esta problemática se realiza desde dos esquemas teórico conceptuales. Por un lado, se revisa la perspectiva del curso de vida y, por el otro, se complementa con la perspectiva de la interseccionalidad con el fin de dar cuenta de las diversas dimensiones y categorías sociales tales como el género, la clase social, el origen étnico y la edad, que impactan sobre el acceso a los recursos y las oportunidades. La aproximación metodológica que se utiliza es el enfoque de los relatos de vida, en donde se exponen ciertos fragmentos de las trayectorias experimentadas que integran una narrativa completa.

Palabras clave: niñas; agencia; violencia; México.

\footnotetext{
${ }^{1}$ La presente publicación es parte de los resultados de la investigación Niñas y trabajo doméstico en el sureste de México, financiada por la Beca a las Mujeres en las Ciencias Sociales 2017 de la Academia Mexicana de Ciencias.

2 Investigadora Cátedras Conacyt Ecosur. Correo electrónico: saraimirandaj@gmail.com
} 


\title{
Being a Girl, Indigenous and Migrant. Life Course and Agency in Social Contexts Marked by Gender Violence and Inequality. The Case of a Tsotsil Girl From The Highlands of Chiapas
}

\begin{abstract}
In Mexico the state of Chiapas has historically shown the greatest social gaps; most of the inhabitants have very little access to opportunities for the achievement of well-being conditions of living, but also experience on a day to day basis persistent discrimination by class, ethnic o genre factors, which contribute to deepening their social vulnerability.This article deals with the trajectory of an indigenous girl from the region of Los Altos de Chiapas who, in a family and community context marked by inequality and violence, has managed to make use of her individual resources to change the course of her life. The general objective is to describe the various strategies that the girl deploys throughout the course of her life as well as to identify the resources that allow her to use the agency to get out of some situations of violence and discrimination. This article's approach is carried out since two theoretical schemes. On the one hand, it uses the perspective of the life course. On the other, this approach is complemented with the intersectionality perspective. The last one gives an account of the different dimensions and social categories such as gender, social class, ethnic origin, and age, that impact on the access to resources and opportunities. Regarding methodological perspectives, this article utilizes the approach of the life stories, exposing fragments of the experienced trajectories to integrate a complete narrative.
\end{abstract}

Keywords: girls; agency; violence; Mexico.

A una niña recién nacida se le toca las manos con los enseres de trabajo de una mujer: el huso, las cardas, los palos del telar, la mano del molcajete y el mecapal para cargar leña. Poco a poco va aprendiendo los trabajos de su madre. A la mujer se le llama Dueña de la casa o la Dueña de la Cama. Muchas veces ya está casada antes de empezar a menstruar. La gran mayoría de las mujeres mayas confiesa que sus padres las casaron o, como dicen ellas, las vendieron a hombres que jamás habían visto antes y que su primera experiencia sexual, llamada en tsotsil la mordida del murciélago, fue en efecto una violación, aunque algunas, andando el tiempo, llegaron a querer a sus maridos. Bastantes mujeres tsotsiles de la nueva generación han decidido no casarse: ¿Para qué voy a aguantar a un borracho que me pega? El papá borracho que pega a su mujer es el tema universal de las canciones de cuna en tsotsil ${ }^{3}$

\section{Introducción}

La niñez y la adolescencia en el mundo enfrenta un sinnúmero de problemáticas tales como la pobreza, la desigualdad, la violencia y la discriminación. La extensión y

\footnotetext{
${ }^{3}$ Dueña de la cama. Ámbar Past.
} 
profundización del modelo económico basado en las economías abiertas y la competencia comercial a escala global ha significado una importante disminución del papel del Estado de Bienestar, con lo que ha disminuido el rol de los estados como proveedores de servicios básicos como la salud y la educación, lo que se traduce en un aumento considerable de población con carencias materiales.

Las niñas, los niños y los adolescentes ${ }^{4}$ son parte de la población considerada como vulnerable frente a las crisis económicas, los incrementos de los niveles de marginación y la profundización de los mecanismos violentos de control social. En México, las estimaciones más recientes dan cuenta de que para el año 2014 uno de cada dos NNA era pobres, en tanto que uno de cada nueve era pobre extremo (CONEVAL y UNICEF, 2014). Estas cifras muestran que la mitad de la población de 0 a 17 años no cuenta con las condiciones básicas para acceder a la alimentación, la salud, la educación y los servicios básicos (CONEVAL y UNICEF, 2014).

Dicha situación se profundiza cuando se trata de NNA que hablan alguna lengua indígena, pues 90.8 por ciento de ellos se situó en condición de pobreza, porcentaje que supera por mucho a quienes no reportan hablar lengua indígena con 60 por ciento. Adicionalmente, se observa una importante diferencia de NNA pobres según región y entidad federativa, las estimaciones del CONEVAL revelan que Chiapas, Oaxaca, Guerrero y Puebla son las entidades con mayor incidencia de niñez y adolescencia en pobreza con 82.3, 72.6 y 72 por ciento respectivamente, frente a Nuevo León (24.9\%) y Baja California (34.9\%) (CONEVAL y UNICEF, 2014). ${ }^{5}$

El estado de Chiapas es la entidad que ha mostrado mayores atrasos sociales desde larga data, la mayoría de la población que habita a lo largo del territorio chiapaneco cuenta con escaso acceso a las condiciones mínimas para el logro del bienestar, pero además experimenta día a día la persistencia de la discriminación por la vía de la clase, el género y la etnia, lo que abona a la profundización de la vulnerabilidad social.

Los niveles de pobreza y los rezagos sociales no se viven de la misma forma en cada grupo social, ni en cada etapa de la vida. Las vivencias de las niñas y las adolescentes adquieren matices ya que, además de la edad, las discriminaciones que las afectan se verán influenciadas según su condición de clase o de origen étnico. Además de las desventajas en

\footnotetext{
${ }^{4}$ En adelante NNA.

${ }^{5}$ Diversos autores relacionan las desigualdades regionales con aspectos de orden económico, cultural, político y social, en los indicadores macroeconómica resaltan los casos de Chiapas, Guerrero, Oaxaca y Tlaxcala como las entidades con el menor PIB per cápita. Contrariamente a los casos del Distrito Federal y Nuevo León como las entidades con el mayor PIB per cápita (Ordoñez, 2015). Al respecto cabe señalar que históricamente los estados del sur tales como Chiapas, Guerrero y Oaxaca han sido las entidades con mayores rezagos sociales, por diferentes factores, entre ellos la mayor presencia de población indígena, en tanto los estados ubicados en el norte, entre ellos Nuevo León y Baja California detentan los mejores niveles de bienestar. Ver figura 1: mapa anexo.
} 
los indicadores de educación y salud, existe conocimiento de algunas condiciones de subordinación a partir del género y la edad que perpetúan la transmisión generacional de desigualdades. Se sabe, por ejemplo, que en las comunidades indígenas hay una práctica extendida de matrimonios forzados que obligan a las niñas y las adolescentes a contraer matrimonio desde cortas edades a cambio de pagos en dinero o en especie (Rosagel, 2014).

Pese a ello, las niñas y las adolescentes toman decisiones y ejercen la agencia. Asimismo, despliegan estrategias para hacer frente a las desigualdades de poder que enfrentan en el día a día. Este artículo versa sobre la trayectoria de una niña indígena tsotsil de la región de los Altos de Chiapas que, en un contexto familiar y comunitario marcado por la desigualdad y la violencia, ha logrado hacer uso de sus recursos individuales para influir en el desarrollo de su vida. El objetivo general es describir las diversas estrategias que la niña despliega a lo largo de su curso de vida e identificar los recursos que le han permitido ejercer la agencia para salir de algunas situaciones de violencia y discriminación.

El acercamiento a esta problemática se realiza desde dos esquemas teórico conceptuales, por un lado, se revisa la perspectiva del curso de vida y por el otro se complementa con la perspectiva de la interseccionalidad con el fin de dar cuenta de las diversas dimensiones y categorías sociales tales como el género, la clase social, el origen étnico y la edad, que impactan sobre el acceso a los recursos y las oportunidades materiales y sobre la forma en que se experimentan las violencias.

La aproximación metodológica que se utiliza es el enfoque de los relatos de vida, en donde se exponen ciertos fragmentos de las trayectorias experimentadas que integran una narrativa completa (Dávila, 2002). Las narrativas de vida en el caso de las niñas y las adolescentes indígenas, aunque cortas, son ejemplos contundentes que posibilitan una lectura e interpretación de aspectos clave para la comprensión de los efectos de las estructuras sociales y la tensión que se experimenta al ejercer la agencia.

Para este análisis, se ha seleccionado la narrativa de una adolescente ${ }^{6}$ indígena tsotsil de 17 años originaria de la región de los Altos de Chiapas. La chica seleccionada migró y se incorporó al trabajo doméstico en la ciudad de San Cristóbal de las Casas a los 12 años de edad. La experiencia de esta adolescente, lejos de que se la considere desde lo único como cualquier discurso particular, se la considera más bien desde su singularidad social en términos de su experiencia como un sujeto con un curso de vida que transita en los intersticios entre la violencia, la migración y el mercado de trabajo local. En este sentido, se entiende "la

\footnotetext{
${ }^{6}$ El título del artículo obedece a que el análisis del curso de vida de la adolescente se realiza a parir de que decidió migrar a los 12 años, es decir, cuando aún era una niña.
} 
singularidad como el cruce entre lo particular de una biografía y lo social, es decir, la forma que toma lo social cuando es apropiado por un individuo" (Lindón, 2003, p. 339).

En realidad, lo que se busca es leer un contexto social que se presenta en este relato pero que podría estar presente en muchos otros casos de niñas y adolescentes indígenas y migrantes. La herramienta de las narrativas brinda la ventaja de que en los casos estudiados y seleccionados es posible entrever un contexto social de sentido, en el que las narrativas particulares seguramente coinciden y están presentes en muchas otras (Lindón, 2003), lo cual aporta rigurosidad metodológica y científica al análisis.

Se parte del reconocimiento de que las niñas y las adolescentes indígenas originarias de la región de los Altos de Chiapas habitan un mundo lleno de dificultades de orden estructural y que sus respuestas y actuar cotidiano responden a una compleja combinación entre lo social y lo individual, pero al mismo tiempo se propone abandonar una postura adultocéntrica que niegue la capacidad individual para practicar y ejercer la agencia y la autonomía de las niñas y las adolescentes.

\section{El curso de vida y las violencias interseccionales}

El papel de la agencia en la vida de las mujeres y niñas indígenas ha sido estudiado por diversos autores y desde diferentes perspectivas. En Latinoamérica, por ejemplo, el foco de los estudios que vinculan el ejercicio de la agencia de las mujeres se ha situado sobre el fenómeno migratorio. A partir de estudios de caso de mujeres que migran del cono sur hacia Europa o mujeres que migran a los principales polos de atracción en Sudamérica se ha puesto en evidencia que las decisiones sobre migrar y movilizarse están mediadas por la capacidad de agenciamiento lo que tiene como resultado la construcción de espacios de asociación para la supervivencia social, económica y personal (Marius Domínguez, 2017).

En algunas investigaciones realizadas con mujeres latinoamericanas que migran hacia España para ejercer tareas domésticas y de cuidados, se observó cómo la agencia opera conformando un conjunto de estrategias de resistencias frente a condiciones estructurales que instalan a dichas mujeres en un lugar de subordinación. En este sentido, las mujeres migrantes encuentran en la subversión y resignificación nuevas formas de convertirse en sujetas activas de transformación (Marius Domínguez, 2017).

Para el caso de la región sur de México, donde hay una preeminencia importante de migración tanto urbano-rural como internacional, así como un importante flujo de población migrante indígena, se han realizado también algunas investigaciones sobre el ejercicio y el significado de la agencia. Investigaciones centradas en la práctica y la negociación como manifestaciones cotidianas de la agencia de mujeres migrantes de Centroamérica, muestran 
cómo, aun en situaciones de profunda vulnerabilidad, las mujeres y las jóvenes que emigran hacia el sur de México desde países como Guatemala, Honduras y el Salvador encuentran distintas formas de poner resistencia o ganar espacios de negociación, sobre todo en empleos vinculados al servicio doméstico y el cuidado de personas enfermas o adultos mayores (Blanco, 2014).

Otras investigaciones, enfocadas en la realidad de mujeres indígenas y campesinas de la región sur de México, han dado cuenta de la heterogeneidad de las agencias femeninas en función de contextos regionales e históricos diferenciados. De este modo, sostienen que la capacidad de acción de las mujeres puede tener lugar en un sinnúmero de espacios, desde la participación política local hasta la participación femenina en religiones organizadas, espacios que tradicionalmente se han percibido como opresores y constrictores de la acción. Como bien afirma Cañas (2018):

las agencias femeninas no siguen trayectorias lineales, sino que se construyen y reconstruyen a partir de articulaciones múltiples. Las mujeres se han vinculado con diferentes opciones religiosas más o menos conservadoras, llevando a la práctica sus discursos y normas religiosas, pero también reinterpretándolos (p. 1651).

En relación con la niñez y la práctica de la agencia, encontramos también estudios que han abordado el tema. Hay dos temáticas fundamentales que no podrían ser entendidas sin la noción de agencia en la niñez: la migración y el trabajo. Al respecto, los estudios recientes de Pavez Soto (2017) han mostrado que, para estudiar los nuevos flujos migratorios, se requiere tomar en cuentan la participación de la infancia considerando que

Las niñas y los niños son "actores sociales con capacidad de agencia" que despliegan relaciones de poder generacionales y de género con los otros actores, en sus familias y los espacios sociales que habitan, conformando una generación social (Pavez Soto, 2017, p. 103).

Para Pavez Soto (2017) la agencia de la niñez se materializa en la toma de decisiones relacionadas con la movilidad, en diferentes circunstancias, ya sea por aquellos que deciden migrar de forma autónoma o no acompañados o quienes en cierta medida intervienen en la movilidad familiar.

La agencia de las niñas y los niños también se ha puesto en evidencia al analizar las dinámicas en que se incorporan al trabajo desde una perspectiva de los sujetos activos. Incluso existen organizaciones sociales que reconocen y luchan por el derecho a la participación política y laboral de los niños y las niñas. Asimismo, rescatan la capacidad de decisión en el contexto laboral, y las ventajas que representa el trabajo para el aprendizaje y empoderamiento de quienes laboran en situaciones adecuadas (Ver los trabajos recientes de Liebel, 2017a). 
En este sentido, el análisis de los fenómenos como la migración, el trabajo infanto adolescente y la violencia contra las niñas y las adolescentes implica dilucidar varios elementos por tratarse de prácticas heterogéneas que se presenta cruzadas por varías dimensiones, desde el género, la etnia y la edad. Este apartado se centra en exponer las bases conceptuales de la perspectiva teórica del curso de vida para encuadrar a la migración y al trabajo infanto adolescente como fenómenos sociodemográficos, pero también como estrategias de acción frente a algunas opresiones vinculadas con la violencia de género y las relacionadas con el origen indígena. Por tanto, se realiza una breve exposición de los elementos más importantes del enfoque del curso de vida y se complementa con la perspectiva de la interseccionalidad para el análisis de las violencias.

Se busca hacer un esfuerzo por poner en el centro del análisis la toma de decisiones de las niñas y las adolescentes y el ejercicio de la agencia, examinándolo como la intersección de condiciones sociales diacrónicas y sincrónicas en un espacio social determinado. Entendidos así, tanto la migración, el trabajo infanto adolescentes y las violencias deben considerarse como prácticas sociales que se cruzan con las relaciones institucionales, comunitarias y familiares.

Bajo este tenor, las niñas y las adolescentes indígenas originarias de los Altos de Chiapas que han migrado y han iniciado su vida laboral a edades tempranas son portadoras de relaciones sociales y de cierto acontecer histórico y geográfico. No es casual que en el sureste mexicano, la región con mayor cantidad de población indígena, siga vigente el modelo colonial de servidumbre basado en diferenciaciones raciales en donde las familias mestizas se atribuyen el papel de educadoras y civilizadoras frente a mujeres, niñas y adolescentes indígenas y pobres, provenientes de contextos rurales imaginadas como "seres carentes, cuyo futuro debe estar ligado necesariamente a la tutela" (Cumes, 2014, p. 48)

Entonces, para comprender los cursos y estrategias de acción de las niñas y las adolescentes indígenas puede ser útil pensarlas a través del análisis de las dinámicas de interjuego entre los individuos y la sociedad, es decir, los vínculos entre el nivel social macro y el micro. Incluso visualizar también el nivel mesosocial como una esfera en el que suceden las prácticas sociales y se encuentran los niveles macro y micro. Al respecto, Przeworsky (1982), quien es un clásico en estas discusiones, afirma que:

las relaciones sociales son dadas así a los individuos como condiciones objetivas de opción. Son independientes de la voluntad individual porque se heredan como consecuencia de acciones anteriores [y]...las acciones de múltiples actores sociales tienen consecuencias para el estado de la sociedad. (p. 73).

Bajo esta premisa, ser una niña o una adolescente indígena significaría tener una estructura de opciones familiares, comunitarias, regionales, que les llevan a optar por la 
migración y el trabajo desde la niñez. Se podrían considerar así, como un ejemplo de que las prácticas de los individuos están determinadas por una estructura de opciones impuesta, que constriñe sus prácticas en cada circunstancia histórica (Martínez, 2014). Para el caso que nos ocupa, una niña o adolescentes afectada por la violencia familiar y la violencia de género, tendrá la opción de migrar y ocupar los tipos de empleos de acuerdo con su condición social, su tiempo histórico y según su estructura de motivaciones.

A partir de lo anterior, se ha decidido utilizar el enfoque del curso de vida. Este enfoque toma en consideración diversos aspectos ya señalados. Se nutre además de otros elementos que complementan la mirada y facilitan la integralidad y profundidad del análisis. Son muchas las ventajas que brinda el enfoque teórico metodológico del curso de vida, sobre todo tratándose de lo que interesa en este artículo: el ejercicio y puesta en práctica de la agencia de las niñas y las adolescentes indígenas frente a las violencias que experimentan.

El enfoque teórico metodológico del curso de vida tiene cuatro premisas básicas que Tuirán (1996) ha resumido claramente:

1) ...el curso de vida es un proceso compuesto por un entretejido de complejos dinamismos; 2) reconoce las relaciones recíprocas entre el individuo y el entorno institucional y social; 3) recupera la historia de los individuos, sus motivos y elecciones personales, y sitúa estos elementos en el centro del análisis; 4) cuestiona los modelos estáticos, enfatizando la enorme "plasticidad humana" y la capacidad que tienen los individuos para modificar sus comportamientos. (p. 23)

En coincidencia con ello, vale la pena señalar que los NNA cuentan con capacidad de agencia, aun cuando la sociedad adulta los lea desde un lugar de superioridad y no los reconozca como sujetos capaces de tomar decisiones y cambiar los cursos de vida que el adultocentrismo ve como "lo mejor" para ellos (Liebel, 2017). Esta percepción de superioridad frente a lo incapaz no está limitada únicamente por la edad, sino que variará en función de las categorías constantemente mencionadas en este texto, como el género y el origen étnico. Ver los trabajos recientes de Liebel (2017) y Elson (1982) sobre el reconocimiento del actuar político de los NNA.

El enfoque del curso de vida constituye toda una construcción teórica y analítica que engloba varios elementos. De ahí que es considerada una de las más completas e integrales perspectivas para el estudio de las trayectorias de los sujetos sociales. En términos generales se compone de tres conceptos básicos: la trayectoria, la transición y el turning point (Blanco, 2011).

Asimismo, estos tres conceptos encuentran sustento en cinco principios básicos: el principio del desarrollo a lo largo del tiempo; el principio de tiempo y lugar; el principio del 
timing; el principio de vidas interconectadas; y, el principio de libre albedrío o agency (Blanco, 2011). Según (Oddone y Gastrón, 2008):

El paradigma del transcurso de la vida abarca el desarrollo individual, en contextos sociales, culturales e históricos cambiantes. Se concentra en las trayectorias de vida individuales, así también como en el engranaje de las carreras de los individuos, a través de sus vidas, con aquellos de la familia y del mundo del trabajo. (p. 3)

Para comprender con mayor claridad la noción de trayectoria frecuentemente se utiliza la metáfora de una carrera, una sucesión de prácticas sociales que idealmente deberían cumplirse para llegar a obtener un producto final. Dichas prácticas tendrían como función ordenar de forma jerárquica el uso del tiempo. Ello estaría vinculado con el dicho popularmente conocido de “lo primero es lo primero" (Oddone y Gastrón, 2008, p. 5).

La noción de trayectoria permite entender el conjunto de procesos que transcurren a lo largo de la vida de los individuos desde que nacen hasta que mueren. Se reconoce que los individuos como seres que cumplen ciclos biológicos, pero al mismo tiempo mantienen una relación con otras dimensiones, sobre todo se reconoce enfáticamente que los individuos son partícipes de un sinnúmero de ambientes sociales que lo preceden pero que al mismo tiempo contribuyen a modificar.

La trayectoria no supone alguna cadena o sucesión de eventos en particular ni determinada celeridad en el proceso del propio tránsito; pero efectivamente el enfoque reconoce que existen mayores o menores probabilidades en el desarrollo de ciertas trayectorias vitales. Las trayectorias pueden abarcar una variedad de ámbitos o dominios (trabajo, escolaridad, vida reproductiva, migración, etc.) que son interdependientes. Por ello, las trayectorias de vida proporcionan una visión dinámica a lo largo de una parte sustancial del curso de vida (Elder y Shanahan, 2006, como se citó en Blanco, 2011).

El enfoque del curso de vida da cuenta de la noción de transición. Este concepto se refiere a cambios de estado, posición o situación, no necesariamente predeterminados o previsibles. Sin embargo, se reconoce que a lo largo de la vida de los sujetos sociales se dan ciertos cambios con mayores probabilidades de ocurrencia.

Los cambios o transiciones varían en función de distintas características, sobre todo en función de la edad biológica, debido a que hoy en día sigue predominando un sistema de expectativas en torno a la edad. El paradigma del curso de vida toma en cuenta también que las expectativas respecto a la edad biológica son variables según ciertas circunstancias culturales y sociales.

El tercer concepto, vinculado con los anteriores, es el de turning point. En términos generales se trata de sucesos y eventos que provocan o generan importantes modificaciones 
en el curso de vida. Incluso en ocasiones podrían ser la causa de cambios y virajes en las direcciones antes previstas de los cursos de vida.Diversos autores conciben al turnning point como cambios a partir de acontecimientos claramente identificables, aunque igualmente podrían tratarse de situaciones subjetivas. Sin embargo, en ambos casos se produce un cambio que supone la intermitencia en una o varias trayectorias vitales.

Para Oddine y Gastron (2008) los turning point pueden relacionarse con transiciones normativas o no normativas y viceversa. Ello dependerá de:

a) Cuando coincide con una crisis o es seguida por una crisis inesperada; b) Cuando una transición normativa es acompañada por un conflicto familiar, siendo el resultado de una asincronía entre las transiciones individuales y colectivas; c) Cuando una transición normativa está fuera de tiempo; d) Cuando una transición normativa es seguida por consecuencias negativas no previstas en la transición; e) Cuando se requiere un ajuste social en relación con una transición normativa diferente. (p. 273)

Las tres nociones hasta ahora señaladas son las herramientas analíticas básicas del enfoque teórico metodológico del curso de vida que “... reflejan la naturaleza temporal de las vidas y captan la idea del movimiento a lo largo de los tiempos históricos y biográficos" (Elder, Kirkpatrick y Crosnoe, 2006, en Blanco, 2011, p. 8,). Por otro lado, el enfoque del curso de vida tiene su soporte en cinco principios básicos y fundamentales, aunados a los conceptos descritos líneas arriba.

El primer principio es el del desarrollo a lo largo del tiempo. Hace referencia a un análisis de largo plazo, debido al reconocimiento explícito de que el desarrollo humano es una seríe de sucesos biológicos que van desde el nacimiento hasta la muerte y que no es posible entender momentos específicos si no se conocen momentos y sucesos precedentes. Implica que "estudiando las vidas a lo largo de períodos sustanciales de tiempo incrementamos el potencial del interjuego entre cambio social y desarrollo individual" (Elder, Kirkpatrick y Crosnoe, 2006, como se citó en Blanco, 2011, p. 11,).

Un segundo principio es el de lugar y tiempo. Hace énfasis sobre lo contextual; establece que el curso de vida de los sujetos sociales "está 'incrustado en' [embedded] y es moldeado por los tiempos históricos y los lugares que le toca experimentar a cada persona" (Elder, Kirkpatrick y Crosnoe, 2006, p.12, como se citó en Blanco, 2011).

Este principio tiene como función incorporar la idea de que las biografías de los individuos se ubican en tiempos históricos y comunidades determinados con las respectivas diferenciaciones por género, por clase social o estrato socioeconómico y por raza o etnia, entre otras (Blanco, 2011). 
El tercer principio es el conocido como el timing. Hace alusión al hecho de que todo individuo tiene una vida que "transcurre en un tiempo que se experimenta como un tiempo interior pero que también se inserta en un tiempo socialmente compartido en un mundo intersubjetivo" (Oddone y Gastrón 2008, p. 2). La edad es la categoría que organiza la secuencia de eventos en la vida de los individuos:

La edad, en efecto, puede ser el marcador de un cierto estadio del desarrollo biológico o psicológico, pero también puede hacer referencia a una cierta posición en la organización social de los roles que corresponden a una determinada edad; y así mismo puede remitir a la pertinencia de una cohorte y a su anclaje específico en la historia de la sociedad (Oddone y Gastrón, 2008, p. 2).

En el principio del timing se reconoce la vida en sociedad de los individuos, el impacto del tiempo social sobre las vidas individuales, y las posibles modificaciones que la historia generaría a nivel de la vida cotidiana de los individuos.

Otros autores ven al timing como el factor que regula la entrada y salida de los individuos entre sus distintos papeles ya sea en la familia, en el trabajo o en la comunidad. Establecen que debido a que el timing se ve afectado por el cambio histórico, tendrá a su vez efectos en el transcurso de la vida en diversos ámbitos: demográfico, institucional, legislativo, y de la estructura económica (Oddone y Gastrón, 2008). El timing es una herramienta para entender la forma en que los individuos aprovechan o no las oportunidades que les proporciona la estructura institucional y social, por ejemplo, las ofertas educativas y laborales (Blanco, 2011).

El cuarto principio es el denominado principio de vida interconectadas (linked lives). Enuncia que las vidas humanas siempre se viven en interdependencia, o sea, en redes de relaciones compartidas, y que es en estas redes donde se expresan las influencias históricosociales. Este principio ayuda a visualizar la interdependencia de las diversas trayectorias de un mismo sujeto frente a otros individuos y grupos (Blanco, 2011).

Finalmente, está el principio del libre albedrío (agency). Subraya que los sujetos sociales no son pasivos, sino que son capaces de elegir y realizar actividades, es decir, construir su curso de vida. El curso de vida reconoce que las elecciones de los sujetos están en cierta medida limitadas por las circunstancias históricas, espaciales y sociales que le toquen a cada individuo (Blanco, 2011).

Los elementos que componen la perspectiva del curso de vida pueden complementarse con una perspectiva interseccional, sobre todo para el análisis de trayectorias marcadas por diferentes tipos de violencias. La noción de interseccionalidad ha cobrado fuerza en las últimas décadas, debido a su utilidad práctica para dar cuenta de las diversas categorías que puede combinarse para generar opresión. Una de las ventajas de 
esta noción es la utilidad para revelar el impacto de ciertas estructuras como el racismo, el clasismo y el adultocentrismo (Duarte, 2012).

Con esta perspectiva se fortalecen los estudios y las investigaciones relacionadas con la desigualdad y la subordinación de colectivos sociales que históricamente han estado en desventaja, tales como las mujeres, los indígenas, los migrantes, la comunidad LGBT, entre otros. Desde este abordaje se muestra una interesante gama de matices a partir del reconocimiento de la interacción simultánea de múltiples ejes de discriminación, ya sea por la vía de la raza, la etnia, la edad, la clase y la orientación sexual (Muñoz, 2011).

Ertürk (2005) aplica el enfoque de la interseccionalidad para el estudio de la violencia contra las mujeres y afirma que:

La vulnerabilidad de las mujeres ante la violencia está relacionada con su posición respecto a múltiples sistemas de desigualdad. Se observa que este estado de vulnerabilidad tiende a incrementarse a medida que estos sistemas se entrelazan, lo que da lugar a diferentes niveles y grados de discriminación y exclusión que variarán en función de los grupos de mujeres. (Erturk, 2005 como se citó en Muñoz, 2011, p. 73)

Para el caso de las niñas y las adolescente indígenas se observa con claridad la opresión por varias vías, primero por la vía del origen étnico, ya que por tradición colonial sigue existiendo la idea de que mujeres y hombres indígenas son inferiores con respecto a ladinos, mestizos o élites raciales; en segundo lugar se enfrentan a la opresión misma del género que se manifiesta en la noción de la inferioridad de las mujeres frente a los hombres; a su vez está la discriminación por la vía de la clase social, ya que en su mayoría estas niñas y adolescentes son pobres y finalmente se enfrentan a la opresión de la edad pues conviven en una sociedad adultocéntrica que percibe a los NNA como seres carentes, en formación y con escasa capacidad de acción. Todo este cúmulo de opresiones y desventajas se traducen en mayor vulnerabilidad frente a la violencia, desde la violencia estructural que limita el acceso al bienestar económico, hasta la violencia física y emocional.

En relación con las opresiones vinculadas con la categoría de edad se han realizado múltiples investigaciones sobre mujeres de la tercera edad, niñas y adolescentes que ven acentuada su vulnerabilidad frente a la violencia y la desigualdad. Ello se profundiza tratándose de niñas y adolescentes con múltiples vías de subordinación, en este caso niñas, indígenas, pobres, migrantes (Chiarotti, 2003 y Bezarés, 2007).

Los estudios sobre tráfico de niñas y adolescentes dan muestra de un vínculo estrecho entre la subordinación de género asociada a la cosificación sexual de las mujeres y las niñas, exponiéndolas a procesos de hiper visibilización como objetos sexuales, e invisibles como sujetos de derechos (Muñoz, 2014). Procesos similares enfrentan las niñas y las adolescentes indígenas, quienes debe cumplir los mandatos tradicionales que se esperan de 
ellas en sus comunidades, por ejemplo, casarse una vez que terminan la educación básica y dedicarse a las actividades domésticas y de cuidados familiares (Vizcarra y Marin, 2006).

De hecho, en las comunidades indígenas la construcción social del género inicia desde tempranas edades y se pone en práctica mediante una marcada división sexual del trabajo, que solo da valor a las mujeres y a las niñas en función de su capacidad reproductiva, concebida como fin natural de la mujer, en tanto que los hombre son los jefes, los dueños de las mujeres, al grado de atribuirse la libertad de venderlas y comprarlas, los que tienen el poder y toman las decisiones sobre la vida de las niñas y las mujeres (Cacho, 2009).

Las niñas indígenas son educadas desde la cuna para barrer, limpiar, lavar, hacer tortillas y cocinar. Actividades con las que se reafirman los roles supuestamente naturales del ser mujer. Los niños también ayudan en actividades del campo u otras tareas fuera del ámbito doméstico. No obstante, los niños al igual que los adultos hombres, son sujetos de otros derechos que a las niñas les son negados, como el derecho a estudiar (Cacho, 2009).

\section{Ser niña, indígena y migrante en la región de los Altos de Chiapas}

Los relatos analizados a continuación corresponden a una adolescente de 17 años de edad, indígena tsotsil originaria de la localidad Chilimjoveltic del municipio de San Juan Chamula. Tanto su localidad como el municipio de origen están clasificados como de muy alto grado de marginación (CONAPO, 2016).

Esperanza $^{7}$ tomó la decisión de migrar hacia la ciudad de San Cristóbal de las Casas en busca de un empleo, pero también como una respuesta a las violencias de género y comunitarias que padeció desde su niñez. Es la tercera hija de siete hermanos(as), tiene una hermana mayor, un hermano varón es el segundo, le sigue Esperanza y las cuatro siguientes menores a ella son mujeres. Todos los miembros de la familia tienen al tsotsil como lengua materna y al castellano como segunda lengua.

Su padre se dedica a las labores del campo y eventualmente trabaja en actividades relacionadas con la construcción como albañil. Su madre trabaja en las actividades domésticas y de cuidados al interior del hogar. La narradora elegida afirma que cuando cursó el quinto año de primaria su padre le dio la orden de abandonar la escuela, ya que pronto cumpliría 12 años y debía ser dada en matrimonio. Ante dicha imposición Esperanza dice haber sentido miedo y enojo, ella quería ser enfermera, deseaba seguir estudiando la secundaria, pero la decisión ya estaba tomada.

\footnotetext{
${ }^{7}$ Este nombre es ficticio, se han omitido los datos que pudieran dar pistas o facilitar su identificación, al igual que la de sus familiares.
} 
Mi papá no quiso de estudiar, dijo que si tienes papá que me compra las enaguas o que me iba a dar para casar y mi marido me va comprar las enaguas. Por eso ya no fui para sexto. Pero yo si quería, pero también dijo mi papá que en el pueblo luego castigan al hombre que no mantiene a sus hijas pues. Y yo no quería el castigo para mi papá, y ya no fui a la escuela. Yo si quería ir, en el otro pueblo hay secundaria, mi hermano fue a la secundaria en el otro pueblo. ${ }^{8}$

Cuando Esperanza abandonó la escuela estuvo unos cuantos meses (no recuerda exactamente el tiempo) en la casa de origen contribuyendo con las tareas domésticas. Expresa que en esa época se sintió triste y aburrida, además dice que siempre pensaba en volver a la escuela primaria. Le entusiasmaba la idea de ir al otro pueblo a la secundaria. Ante la insistencia de su parte para volver, su madre la reprendió con golpes en varias ocasiones.

Por lo general, los NNA que habitan en contextos rurales y de alta marginación no cuentan con la oferta educativa suficiente, la lejanía puede representar un gasto excesivo que se acentúa mucho más en las niñas, ya que frente al papel histórico que han de cumplir, la escolaridad es una pérdida de tiempo y una traba para los procesos de reproducción cotidiana de las familias (Hoyos, 2000), tal como lo evidencia el caso de la narradora.

La literatura especializada sobre el rol de las niñas en las comunidades indígenas muestra los rezagos en materia educativa que tienen frente a los niños. Se valora mucho más invertir en la escolaridad de los varones que en la de las niñas. Para ellas el destino manifiesto es el matrimonio y la maternidad (ONU Mujeres, 2018). En Chiapas para el año 2015 el promedio de años de escolaridad para las mujeres de 15 años y más fue de 6.6 años mientras que para los hombres de la misma edad fue de 7.6 años (INEGI, 2015). Es decir, una brecha de un año entre hombres y mujeres.

Asimismo, Chiapas es una de las entidades federativas con mayor tasa de matrimonios infantiles, de cada 100 matrimonios 15 son de menores de edad (ONU Mujeres, 2018). En este sentido, la chica que aporta su experiencia narrativa tiene también algo que decir. Su padre le afirmó que había encontrado un esposo para ella:

En ese tiempo lo que menos me gustó fue que mi papá me buscó un marido. Yo no quería casarme y estar como mi hermana, que le pega y le grita el hombre, los maridos pegan y gritan y son borrachos, no quería como mi hermana. Y si le decía eso mi papá se enoja. Pero yo no quería casarme.

Si se analizan estos fragmentos a la luz de la perspectiva del curso de vida, se observa con claridad cómo se cumple el hecho de que la trayectoria de esta chica se entrelaza

\footnotetext{
${ }^{8}$ Los relatos que se presentan son transcripciones literales de los relatos obtenidos en las entrevistas. El idioma materno de la relatora no es el castellano, por lo que sus expresiones podrían resultar diferentes al castellano conocido en el mundo de habla hispana.
} 
con otras trayectorias vitales, sobre todo en relación con su familia de origen y su padre. Pues los castigos que se dan en la comunidad a los hombres que no muestran ser proveedores y las tradiciones asociadas al lugar de las mujeres en el matrimonio determinaron que Esperanza saliera de la escuela y que su padre se diera a la tarea de encontrar un candidato para esposo de su hija.

Como bien puede observarse en el relato, la niña que es la protagonista de esta historia tiene una trayectoria moldeada por un tiempo histórico y un lugar determinado, es decir, le ha tocado atravesar la niñez en una comunidad rural, que registra altos niveles de marginación, en una de las entidades federativas con mayores rezagos de infraestructura educativa. Esta situación correspondería al segundo principio del tiempo y el lugar de la perspectiva del curso de vida.

También es posible aplicar el principio de timing, ya que alude a que la vida de los sujetos transcurre en un tiempo interior y un tiempo socialmente compartido. La edad y las transiciones que de esta categoría se derivan son un ejemplo de ello. Para las chicas indígenas de la región de los Altos de Chiapas la entrada en la pubertad o la adolescencia es un marcador para entrar a otro estadio y asumir el rol de mujeres casadas, de ahí la importancia de buscar un marido o concertar las uniones por parte de los familiares.

El principio del timing también ayuda a entender cómo, a pesar de que en la comunidad de Esperanza existe una escuela a nivel primaria, no se aprovechó hasta el sexto grado y tuvo que abandonar su escolaridad en el quinto año, una vez que cumplió los 12 años de edad. Como afirma Blanco (2011) el timing reconoce la vida en sociedad de los individuos y es una herramienta para entender la forma en que estos aprovechan o no las oportunidades que les proporciona la estructura institucional y social, aunque sea precaria y escasa, como lo es la oferta educativa en los contextos rurales del estado de Chiapas. En este sentido se cumpliría la premisa de que el principio del timing tiene efectos en el transcurso de la vida en diferentes ámbitos como el demográfico, el institucional y en la estructura económica. Esperanza, al no completar la escolaridad primaria, forma parte de la estadística que pone en desventaja educativa a las niñas frente a los niños que se mencionó líneas arriba.

Hasta ahora, con los fragmentos que se ha presentado, pareciera que Esperanza estuvo completamente constreñida frente a las decisiones de los adultos, al tiempo social en el que le tocó nacer y a las tradiciones impuestas históricamente. No obstante, y como bien se marca en el enfoque del curso de vida, los sujetos sociales no son pasivos, tienen capacidad de elegir y de poner en práctica estrategias para sortear situaciones y construir su curso de vida. 
Esperanza, a los 13 años de edad, con toda la estructura social y comunitaria a cuestas, decide migrar hacia San Cristóbal de las Casas en busca de un empleo, pero también tratando de evadir el matrimonio y tratando de salir de la violencia familiar que experimentó en su hogar.

Yo me vine porque un problema que tuvimos en mi casa, mi papá pegó a mi mamá, y nos asustamos, yo me asusté. Mi papá pegó y quitó el dinero a mi mamá, y eso también porque me dio miedo de que estaba buscando un marido. Y ese mismo día tomé una combi, no, dos combis y vine con mi tía que vive aquí. Se enojó mucho mi papá, pero yo quiero trabajar, quiero comprar mi enagua y comprar más cosas y no quería casar. No es lo mismo que yo me lo compré, tengo mi hermana más grande, pero ella nunca tuvo valor de decirle a mis papás que quiero trabajar, yo soy la única que salí más así, quiera o no quiera mi papá yo voy a trabajar, no quiero depender de mi papá, ni del marido, si un día se muere mi papá quién me va a mantener.

Por otra parte, la perspectiva del curso de vida reconoce las limitaciones que imponen las circunstancias estructurales a los individuos, y pone de manifiesto que los individuos se mueven de un lugar a otro a lo largo de sus trayectorias de vida dentro de límites en las oportunidades que ofrece el Estado, el mercado y las instituciones. El siguiente fragmento da muestra de ello:

En San Cristóbal al principio no encontré rápido, y llegué sin saber a dónde llegar, si tenía dinero pero no sabía bien a dónde ir, tenía 13 años, la cosa que yo llegué y me dijeron una señora en el mercado que rentaba un cuarto y así ya no estaba con mi tía, para que no se enoje ella también, cuando llegué se estaba enojando por el gas, y así en el mercado una señora me dijo: "oye ¿no quieres trabajar de lavatrastes?", porque así me dijo la señora porque ella no sabía si yo ya sabía cocinar, y de ahí de lavatraste yo veía cómo le hacían la otras en la cocina y le pregunté a la señora que si ya me podría entrar en la cocina, y le dije: "dame una semana pa que vean cómo yo cocino y ya en una semana si no sé cocinar mejor me voy" le dije. Y la señora como que no quería y me quedé en lavatrastes y ahí pagaban casi mitad y eso no me gustaba.

Esperanza logró salir de un contexto familiar y comunitario de violencia, tomó una decisión, la ejecutó y cambió el curso de vida que su familia tenía pensado y planificado para ella. No obstante, la estructura de los mercados de trabajo, segmentados, informales y precarizados le impidieron incorporarse a un trabajo que ella deseaba. Igualmente, la red familiar con la que contaba no fue suficiente para contenerla, y debió buscar una habitación para rentar.

Una vez en la ciudad Esperanza se enfrentó a diversas discriminaciones tal como lo afirma en las siguientes líneas:

En el mercado de lavatrastes no me gustaba, pero tenía que ir, se levanta a las 4 y media y se empieza a las cinco para servir los cafés, y tener todo limpio. Y yo era de lavatrastes pero la señora me decía de llevar las comidas y los desayunos a los puestos y las bodegas y no me gusta. Eran groseros unos muchachos me decían de tener cosas conmigo. Uno me tiró 


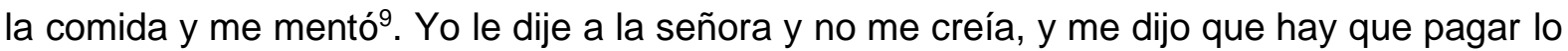
que se cayó, pero fueron esos hombres de las bodegas. Yo por eso no me gustaba salir con las comidas.

Las condiciones laborales que enfrentó Esperanza son el resultado de las estructuras de segmentación e informalidad laboral combinadas con la presencia de violencia vinculada a su género en el espacio público. El bajo salario que no rendía para soportar los gastos cotidianos, más los tratos que recibía de los clientes y de la empleadora la empujaron a volver a su comunidad:

Cuando cumplí 15 mejor me fui a mi casa, la patrona no la aguantaba, no era buena persona, un día me dijo que soy tonta, otro día me pagó poco no lo que decía, otro día me dijo de que soy tonta, otro día no pagó todo por la comida que se cayó de los hombres que me molestaron en las bodegas. Mejor me fui para mi casa y llegué con mi mamá. Mi papá enojó mucho pero no tanto. Y me dijo de buscar un marido. Yo vi el marido que era un muchacho y acepté.

Como apunta el enfoque del curso de vida, las trayectorias son flexibles y no son lineales, puede presentar ires y venires. Tal como le sucedió a Esperanza quien a los 15 años volvió a su comunidad y aceptó contraer matrimonio. En este lapso del curso de vida de la protagonista, se aplica el concepto de turning point. Volver a su comunidad y casarse dio un viraje importante al curso de su vida. Estos cambios que Esperanza eligió cambiaron su vida y su dinámica de forma sustancial. En sus palabras queda muy claro:

De que me casé me fui con mi suegra y ahí estaba también en la casa, eso me aburría y al principio mi marido no tomaba, pero después si empezó a tomar y a tomar, y me pegó. Eso me hizo pensar de que yo me había ido para no estar como mi mamá y mi hermana, pero como en mi pueblo te dicen: ya tienes marido vas a estar con él, o te castigan, te meten a la cárcel si no estás con tu marido. Por eso me fui otra vez (risas), yo no soy como mi hermana, me fui a Puebla, enojó mi papá y mi marido, pero yo quería irme a aprender cocina china, y mi prima que vive en Puebla me ayudó con una señora de restaurant de comida de Corea, y ahí fui otra vez en combi para Tuxtla, de Tuxtla a Veracruz y de Veracruz a Puebla (risas). Y Puebla me gustaba mucho, ahí no escuchaba los chismes de mi pueblo de que la mujer debe estar con su marido (risas).

En el relato se vuelve a observar la agencia que es capaz de ejercer Esperanza. Se repite a lo largo de su curso de vida la constante de la interacción con otros actores sociales y las elecciones que va tomando en función de los sucesos que está experimentando.

Cabe señalar que a lo largo de los procesos que ha experimentado la protagonista, se observa la interacción con diversos ambientes sociales que la precedían pero que, al mismo tiempo, ha contribuido a modificar. Sus hermanas menores ahora han salido de su

\footnotetext{
${ }^{9}$ Expresión que hace referencia a mentar la madre, o bien, decirle groserías
} 
comunidad ya que no quieren seguir con el mandato del matrimonio. Su padre ha apoyado la salida de sus hermanas:

Mis dos hermanas ya vieron conmigo que si no aprenden a ganar la vida y comprar sus enaguas pues, ni modos, a casarse y estar con esos hombres que luego emborrachan y pegan. Ya son más valientes ellas (risas), ahora si están con un trabajo y aprendiendo para tener su propio dinero. Yo siempre les quiero dar el consejo de no casar y de saber ganar la vida. No está muy bien estar con un marido que pega.

Se observa que Esperanza ha transitado algunos periodos en los que se incrementa el potencial de cambio social y de desarrollo individual. Actualmente Esperanza trabaja en la Ciudad de San Cristóbal de las Casas cuidando a una bebé de un año. Volvió de Puebla al sentir que la patrona se enojaba mucho y la regañaba como en sus primeras experiencias laborales. Decidió no volver más con su esposo. No sabe qué va a pasar en el futuro, pero tiene claro que no quiere volver a depender económicamente ni de su papá ni de su esposo. Ha cumplido 17 años. Se siente orgullosa porque sabe cocinar y cuidar muy bien de la niña. Además, está ahorrando para un posible viaje a Puebla, le gustaría visitar a las amigas que conoció en esa ciudad.

Una apreciación importante que sale a la luz en la experiencia de vida de Esperanza es su constante exposición a diversos tipos de violencia. Como se expuso líneas arriba, en su existencia se interseccionan diversas dimensiones que la hacen vulnerable a las violencias. Primero la violencia estructural que enfrentan en su mayoría las niñas y las adolescentes indígenas, que viven en condiciones de pobreza y con escaso acceso a la educación. A ello se suma la subordinación de género que las obliga al cumplir los mandatos del matrimonio y la maternidad, y finalmente la discriminación por la vía de la edad que hace que se decida por ellas sin considerar su opinión: todas esas opresiones y violencias que ha experimentado Esperanza, incluido el acoso y la violencia que sufrió en el ambiente laboral del mercado. Sin embargo, ha ejercido también su capacidad de acción y con ello ha logrado influir y cambiar su curso de vida y su entorno más inmediato.

\section{Reflexiones finales}

A lo largo del este artículo se ha intentado ilustrar dos procesos diferenciados pero que, al combinarse, derivan en una compleja interacción de los sujetos sociales con su entorno social, teniendo en el medio la capacidad de agencia.

Por un lado, el análisis del curso de vida de una niña indígena tztozil que vio en la migración y el trabajo una salida a situaciones de violencia de género; por otra parte, el 
reconocimiento de las diferentes categorías de opresión que derivan en la experiencia de la violencia de manera interseccional.

Como bien lo ejemplifican los relatos expuestos líneas arriba, la chica se encuentra en una situación de vulnerabilidad y expuesta a diferentes tipos de violencia que experimenta a partir de su género, su condición de clase, su origen indígena y su edad. Ha nacido en un contexto local y regional marcado por bajos niveles de bienestar y escasa oferta educativa. Su condición de indígena la coloca en un sector de la población que históricamente se ha incorporado a empleos relacionados con tareas domésticas y de cuidados, con condiciones de informalidad y expuesta a malos tratos y abusos por parte de los empleadores.

En este sentido, varias tensiones se han observado en los relatos que aporta la protagonista de este análisis. Su curso de vida, aunque corto, muestra un entretejido de relaciones complejas y dinámicas, por ejemplo con su padre y las tradiciones comunitarias que dictan el destino de las niñas como esposas y seres en dependencia de los hombres (padre y esposo). Igualmente se lee entre líneas que su curso de vida está relacionado con un entorno institucional, este sería el caso de los mercados de trabajo informales además del Estado con su insuficiencia para garantizar la escolaridad en cada comunidad y para garantizar la protección de sus derechos básicos.

Asimismo, otros elementos de la perspectiva teórica del curso de vida pueden identificarse. El centro de interés en este artículo es claro en su narrativa. La exposición de sus motivaciones personales y sus elecciones. Esperanza estuvo siempre motivada por no ser dependiente de su padre y no seguir el mismo modelo de vida de su madre y hermana mayor. Tomó la decisión de migrar, de trabajar, de volver a su comunidad y de migrar nuevamente.

Pero sin duda lo que sale a la luz de forma sustancial es la capacidad de modificar su ambiente. La influencia que ejerció sobre las hermanas menores no es un aspecto mínimo. Según la narradora, sus hermanas menores ya están eligiendo también otros cursos de vida e intentan salir del mandato histórico que pesa sobre las niñas y las mujeres indígenas.

Con este reconocimiento no se pretende minimizar la importancia de las estructuras sociales ni mucho menos justificar y suavizar los efectos de las violencias de que son objeto las niñas indígenas; pero al reconocer la agencia y la capacidad de acción se evita ver a los NNA desde una postura protectora y compasiva frente a sujetos sociales con el derecho a elegir sobre su curso de vida. Por eso mismo surge una importante interrogante: ¿En dónde debe estar el foco de atención para estas niñas y adolescentes que tienen expectativas y motivaciones diferentes a la impuestas por la costumbre? ¿Cómo podrían generarse los mecanismos de acompañamiento para estas niñas y adolescentes? $\mathrm{Y}$, evidentemente, 
reflexionar y actuar sobre la deuda histórica que mantiene la sociedad mexicana frente a las niñas y las adolescentes indígenas que experimentan múltiples subordinaciones, en una sociedad patriarcal, racista, con niveles alarmantes de desigualdad y de violencia.

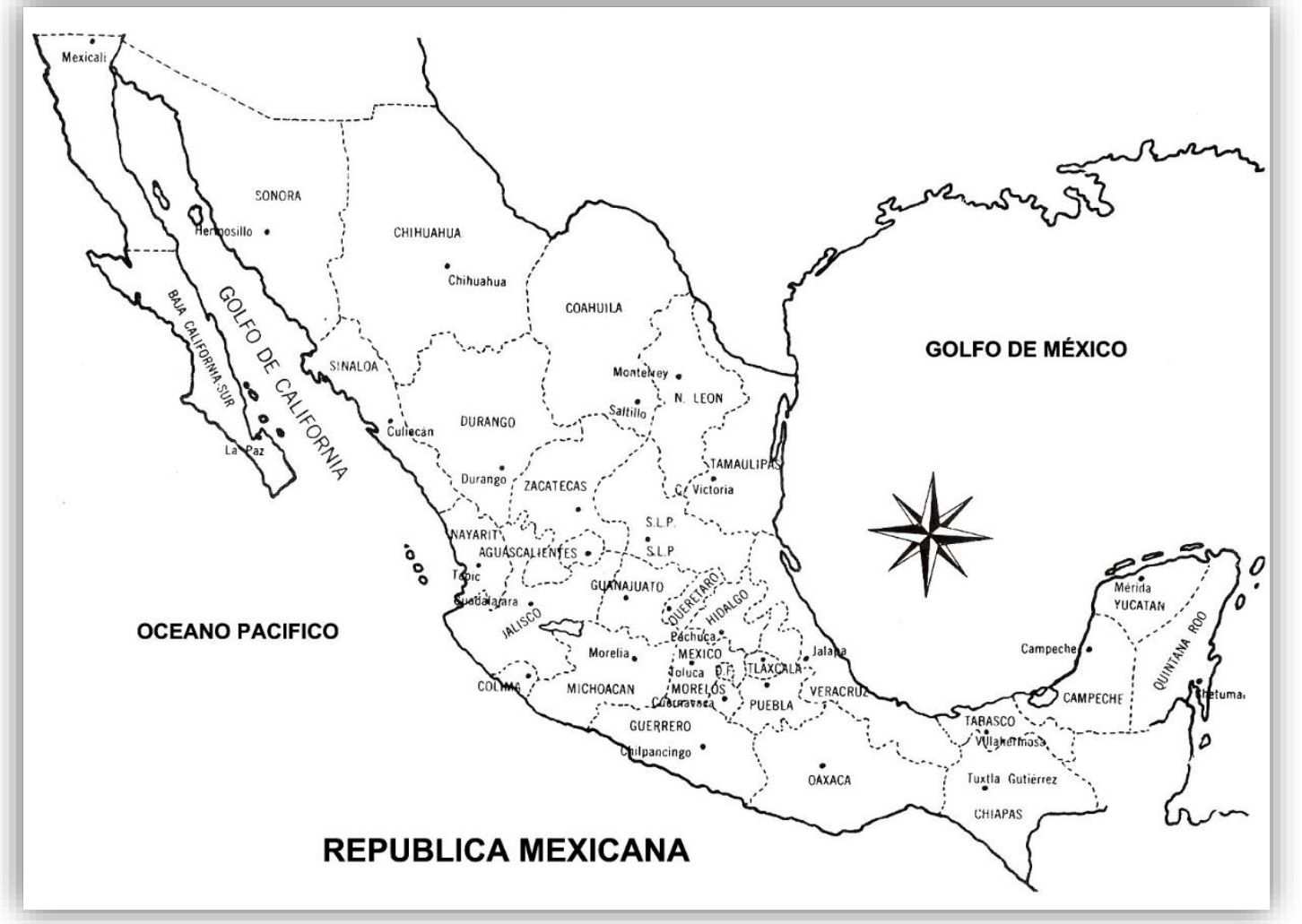

Figura 1. Mapa de Estados Unidos Mexicanos por entidad federativa

Fuente: INEGI, 2018

\section{Referencias bibliográficas}

Bezarés, P. (2007). Aproximaciones para el análisis y el estudio sobre la situación de las mujeres indígenas y migración en Guatemala. Migraciones indígenas en las Américas. (pp. 115-128). San José de Costa Rica: Instituto Interamericano de Derechos Humanos. Recuperado de https://www.iidh.ed.cr/IIDH/media/2031/migraciones-indigenas-en-las-americas2007.pdf

Blanco, B. (2014). Negociación y resistencia: relaciones diádicas en el empleo doméstico de mujeres guatemaltecas en Tapachula, Chiapas. En S. Durin, M.E. De la O y S. Bastos (Coords.) Trabajadoras en la sombra, dimensiones del servicio doméstico latinoamericano. (pp. 201-230). Ciudad de México: Publicaciones de la Casa Chata. 
Blanco, M. (2011). "El enfoque del curso de vida: orígenes y desarrollo". Revista Latinoamericana de Población, año 5, núm. 8, enero/julio, 5-31.

Cacho, N. (2009). La triple opresión. La mujer indígena y campesina en Chiapas y México. Chiapas, México: Centro de Investigaciones Económicas y Políticas de Acción Comunitaria. México.

Cañas, S. (2018). Las mujeres Indígenas y Campesinas del Sureste Mexicano: Agencia Femenina a Debate. Géneros, multidisciplinary journal of gender studies. 7 (2), 1634-1656.

Chiarotti, S. (2003). Trata de mujeres: Sus conexiones y desconexiones con la migración y los derechos humanos. Recuperado de: https://publications.iadb.org/handle/11319/369

Cumes, A. (2014). La "india" como "sirvienta": Servidumbre doméstica, colonialismo y patriarcado en Guatemala. (Tesis de Doctorado en Antropología). México: CIESAS.

Dávila, O. (2002). Biografías y trayectorias juveniles. Última Década, 17, 107-123.

Elson, D. (1982). The Diference on of Children's Labour in the Capitalist Labour Market. Development and Change, 13, 479-497.

Hoyos, S. (2000). ¿Y quién la mandó a ser niña? El trabajo infantil doméstico desde una perspectiva de género. En G. Acosta, E. García y S. Hoyos (coords.). Trabajo infantil doméstico ¿Y quién la mandó a ser niña? Santa Fe de Bogotá: Fondo de las Naciones Unidas para la Infancia (UNICEF) / TM Editores, 109-137.

Liebel, M. (2017), Infancias poscoloniales. Revista Internacional NATS desde los niños, niñas y adolescentes trabajadores. XXI (27), 98-114.

Liebel, M. (2017a). Explotación económica de niñas y niños. Un ensayo teórico para una praxis orientada en el sujeto. Capacidad de Resiliencia. Revista Internacional NATS desde los niños, niñas y adolescentes trabajadores. XXI (27), 49-73.

Lindón, A. (2003), La precariedad laboral como experiencia través de la narrativa de vida. Gaceta Laboral. 9 (3), 333-352.

Marius Dominguez, P. (2017). Agencia femenina en los procesos migratorios internacionales: Una aproximación epistemológica. Revista de Metodología de Ciencias Sociales. 37, 75-99.

Martínez, G. (2014). Chiapas: cambio social, migración y curso de vida. Revista Mexicana de Sociología 76 (3), 347-382.

Muñoz, P. (2011). Violencias Interseccionales. Debates Feministas y Marcos Teóricos en el tema de Pobreza y Violencia contra las Mujeres en Latinoamérica. Honduras: Central America Women's Network (CAWN). 
Oddone, M. y Gastron, B. (2008). Reflexiones en torno al tiempo y el paradigma del curso de vida. Perspectivas en Psicología. Revista de Psicología y Ciencias Afines. 5 (2), 67-83.

Ordóñez, J. (2015). Desigualdades regionales en México: la importancia de considerar las capacidades regionales para el diseño de la política de desarrollo regional. Circunstancia. XIII (36), 25-42.

Pavez Soto, I. (2017). La niñez en las migraciones globales: perspectivas teóricas para analizar su participación. Tla-melaua. Revista de ciencias sociales. 10 (41), 96113.

Przeworsky, A. (1982). La teoría sociológica y el estudio de la población: reflexiones sobre los trabajos de la comisión de población y desarrollo del clacso. (pp.: 59-99). Reflexiones teórico-metodológicas sobre investigaciones en población. Ciudad de México: Consejo Latinoamericano de Ciencias Sociales/El Colegio de México.

Rosagel, S. (17 de marzo de 2014). La venta de niñas y las bodas arregladas son comunes en Chiapas, Recuperado de http://www.sinembargo.mx/17-03-2014/933729.

Tuirán, R. (1996). Transición de la adolescencia a la edad adulta en México. En C. Welti (coord.), Dinámica Demográfica y Cambio Social, Ciudad de México: Fondo de Población de las Naciones Unidas/The MacArthur Foundation/IIS-UNAM, 173185.

Vizcarra, I. y Marin, N. (2006). Las niñas a la casa y los niños a la milpa: la construcción social de la infancia mazahua. Convergencia. Revista de Ciencias Sociales. 40, 39-67.

\section{Otros documentos consultados}

Consejo Nacional de Población. (2016). Datos Abiertos del Índice de Marginación. Recuperado

de http://www.conapo.gob.mx/en/CONAPO/Datos_Abiertos_del_Indice_de_Margin acion.

Consejo Nacional de Evaluación de Política Social y Fondo de las Naciones Unidas para la Infancia. (2014). Pobreza y derechos sociales de niñas, niños y adolescentes en México. Ciudad de México: CONEVAL.

Instituto Nacional de Estadística e Informática. (2015). Encuesta Nacional de Ocupación y Empleo. Recuperado de:

http://www.beta.inegi.org.mx/proyectos/enchogares/regulares/enoe/

ONU Mujeres (2018). Mujeres y niñas rurales. Recuperado de http://www.unwomen.org/es/digital-library/multimedia/2018/2/infographic-ruralwomen. 